\title{
Actitud de estudiantes y profesores del Departamento de Pediatría hacia las tecnologías de la información y comunicación (TICs)
}

Attitude of students and teachers of the Department of Pediatrics towards information and communication technology (ICT)

\author{
Luis Américo Reátegui Guzmán ${ }^{1, a}$, Manuel Hernán Izaguirre Sotomayor ${ }^{1, b}$, Hallder \\ Mori Ramírez ${ }^{1, b}$, Raquel Susana Castro Tamayo ${ }^{1, c}$, Nattaly Aguedo Sussan ${ }^{1, c}$ \\ Facultad de Medicina San Fernando, Universidad Nacional Mayor de San Marcos, Lima, Perú. \\ ${ }^{a}$ Profesor Asociado, Departamento de Pediatria; ${ }^{b}$ Profesor Principal, Departamento de Pediatria; ${ }^{\text {C }}$ Estudiante.
}

\begin{abstract}
Resumen
Introducción. Las tecnologías de la información y comunicación (TICS) están influyendo decididamente en el ámbito educativo, dado que es una herramienta de acceso a gran cantidad de información; sin embargo, su uso en el proceso enseñanza aprendizaje en las instituciones educativas no ha sido significativo. Objetivo. Conocer la actitud de los estudiantes y profesores hacia el uso de las nuevas tecnologías de la información y comunicación Diseño. Investigación no experimental, transversal, de tipo descriptivo y correlacional, de diseño cuantitativo. Lugar. Departamento de Pediatría, Facultad de Medicina San Fernando, Universidad Nacional Mayor de San Marcos, Lima, Perú. Participantes. Estudiantes y docentes. Intervenciones. Aplicación de la Escala de Likert. Se utilizó el análisis de la estadística descriptiva, distribución de frecuencias y medidas de tendencia central. Resultados. La actitud de los docentes hacia las TICs fue más favorable (86\%) que la de los estudiantes (76\%). Conclusiones. La actitud y la satisfacción media global de los docentes del Departamento de Pediatría hacia las TICs fue más favorable que la de los estudiantes, mientras que la actitud muy favorable hacia las TICs fue solo para el quinto de los estudiantes y para el tercio de los docentes. Se espera que la presente investigación contribuya a la mejora de la toma de decisiones frente el uso de las TICs, del proceso enseñanza aprendizaje y de la actitud de los alumnos hacia el uso de las TICS.

Palabras clave. Tecnologias de información y comunicación - TICs; Actitud.
\end{abstract}

\section{Abstract}

Introduction: Information and communication technology (ICT) has influence in education as it is an access tool to wealth of information. Though use in the teaching-learning process in educational institutions has not been significant. Objective: To determine attitude of students and teachers to use of ICT. Design: Non-experimental cross-sectional, correlational, quantitative study. Setting: Department of Pediatrics, School of Medicine, Universidad Nacional Mayor de San Marcos, Lima, Peru. Participants: Students and teachers. Interventions: Likert scale was implemented. Analysis of descriptive statistics, frequency distributions and central tendency measures were used. Results: Teachers attitude towards ICT was more favourable (86\%) compared to students' (76\%). Conclusions: Teachers attitude to ICT was more favourable than students'. It is expected that this study will contribute to improvement in ICT-use decision, teachinglearning process and students' attitude towards use of ICT.

Keywords: Information and communication technology - ICT; Attitude.

An Fac med. 2015;76(3):261-4 / http://dx.doi.org/10.15381/anales.v76i3.11236

\section{INTRODUCCIÓN}

La globalización de las comunicaciones ha evidenciado la necesidad de conocer cuál es la actitud de los estudiantes y docentes del Departamento de Pediatría de la Facultad de Medicina de San Fernando de la Universidad Nacional Mayor de San Marcos hacia las tecnologías de la información y la comunicación (TICs) y a su vez averiguar las necesidades de conocimientos y capacidades que demanda la utilización de dichas tecnologías en el ámbito del ejercicio de la profesión médica ${ }^{(1)}$.

Es por todos conocido que la incidencia del uso de la tecnología en la sociedad en general y en la formación y ejercicio profesional médico en particular ha derivado en reconocer su importante rol en la sociedad del conocimiento en que vivimos ${ }^{(1,2)}$. En consecuencia, el uso de las TICs está influyendo decididamente en el ámbito educativo, dado que es una herra- mienta de acceso a gran cantidad de información. Sin embargo, su uso en las instituciones educativas para la mejora del proceso enseñanza aprendizaje no ha sido significativo ${ }^{(3,4)}$.

En este sentido, la UNESCO enfatiza que "las tecnologías de la información y la comunicación (TIC) pueden contribuir al acceso universal a la educación, la igualdad en la instrucción, el ejercicio de la enseñanza y el aprendizaje de calidad y el desarrollo profesional 
de los docentes, así como a la gestión, dirección y administración más eficientes del sistema educativo" (5).

Por lo tanto, es indispensable que el profesorado de los centros educativos, reciba urgentemente una 'alfabetización digital' y "una actualización didáctica que le ayude a conocer, dominar e integrar los instrumentos tecnológicos y los nuevos elementos culturales en general en su práctica docente" ${ }^{(6)}$.

Por consiguiente, todos los actores involucrados en el proceso enseñanza aprendizaje necesitan la implementación de programas de formación y perfeccionamiento, en donde las tecnologías sean "un medio más, no el fin último, generando metodologías diversas, transformando las estructuras organizativas y generando dinámicas de motivación, el cambio hacia un uso crítico, didáctico y pedagógico de las tecnologías ${ }^{(7)}$.

\section{MÉTODOLOGÍA}

La investigación fue no experimental, transversal, de tipo descriptivo y correlacional, de diseño cuantitativo. La muestra estuvo conformada por 105 estudiantes y por 45 docentes del Departamento de Pediatría entre los meses de enero a diciembre del 2013.

Las unidades de análisis estuvieron conformadas por los estudiantes y por los docentes del Departamento de Pediatría.

Al no haberse utilizado métodos invasivos que pusieran en peligro la salud física y mental de los estudiantes y docentes, no existieron problemas éticos en el presente estudio. Sin embargo, se respetó los principios éticos aceptados por la sociedad y los Comités de Ética de la universidad, así como la propiedad intelectual. La identidad de los estudiantes y de los profesores fue reemplazada por códigos al procesar y reportar los resultados.

El instrumento que se utilizó fue la Escala de Likert, la cual ha sido ampliamente empleada para evaluar la actitud de las personas en el Perú y el mundo. Fueron determinadas las actitudes tanto de estudiantes como de profesores frente a 21 constructos relacionados con las tecnologías de la comunicación e información (TICs).

La técnica utilizada fue la encuesta directa cara a cara. El instrumento utilizado para el recojo de los datos fue Escala de Likert que fue aplicado por un encuestador ajeno al servicio y capacitado en el manejo y procedimiento de su aplicación.

Los datos obtenidos fueron ingresados en Microsoft Office Excel 2000 y tabulados mediante software SPSS versión 15. Finalmente, se realizó el análisis descriptivo de todas las variables, a través de la determinación de proporciones de las variables sociodemográficas y de los 21 constructos mencionados.

En la ejecución de la investigación no se presentaron limitaciones importantes al desarrollo del mismo, salvo las inasistencias de los estudiantes al momento de responder la prueba y la ne- gativa de los docentes a responderla, lo que determinó la exclusión del estudio de varios estudiantes y docentes.

\section{RESULTADOS}

La actitud más favorable hacia las TICs le correspondió a los docentes del sexo femenino, mayores de 51 años, nombradas, con 21 a 30 años de experiencia, comparada con la de los estudiantes de sexo femenino, de 30 a más años de edad y con 11 a más años de permanencia en la universidad.

Así mismo la actitud menos favorable le correspondió a los docentes hombres de 36 a 50 años, contratados, que tenían 1 a 20 años de experiencia, mientras que la actitud indiferente les correspondió a las estudiantes de sexo masculino, de 20 a 29 años de edad y con 1 a 10 años de permanencia en la universidad.

La actitud de los docentes hacia las TICs fue favorable y muy favorable para el $86 \%$, mientras que para los es-

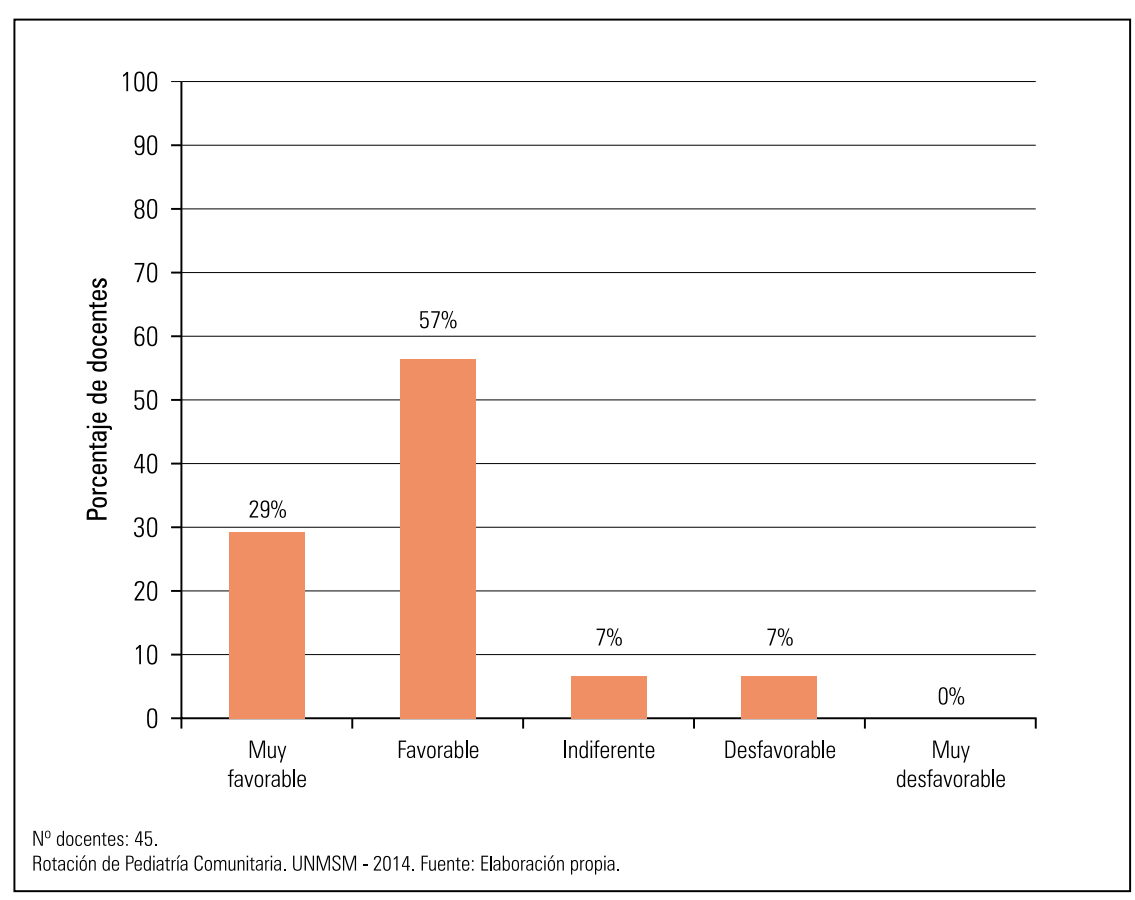

Figura 1. Actitud hacia las TICs de los docentes de la rotación de Pediatria Comunitaria de la Facultad de Medicina. 


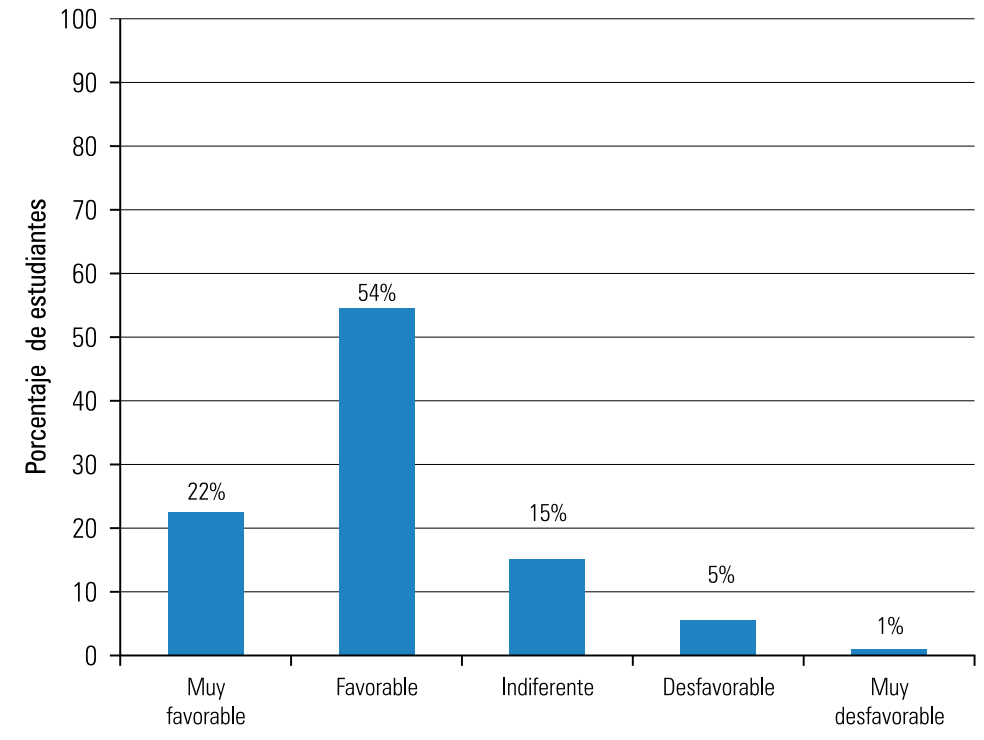

No estudiantes: 105.

Rotación de Pediatría Comunitaria. UNMSM - 2014. Fuente: Elaboración propia.

Figura 2. Actitud hacia las TICs de los estudiantes de la rotación de Pediatría Comunitaria de la Facultad de Medicina.

tudiantes fue en $76 \%$. Contrariamente, $14 \%$ de los docentes tuvo una actitud indiferente, desfavorable o muy desfavorable hacia las TICs, comparada con la actitud de los estudiantes en $21 \%$ (figuras 1 y 2 ).

\section{DISCUSIÓN}

La presente investigación reveló que la actitud de los docentes hacia las TICs fue para el $86 \%$ favorable y muy favorable, demostrando el interés y la predisposición hacia la utilización de la tecnología de punta en provecho del proceso enseñanza aprendizaje. En los estudiantes, el $76 \%$ tuvo una actitud favorable o muy favorable, y más de la quinta parte fue indiferente o tuvo una actitud desfavorable o muy desfavorable hacia las TICs, lo que se constata a diario en las aulas cuando el docente desarrolla las sesiones de aprendizaje. Creemos que la actitud favorable debería ser aprovechada para realizar programas de capacitación para la utilización de las TICs durante el proceso enseñanza aprendizaje. Por otro lado, la existencia de una quinta parte de estudiantes con actitud desfavorable a las TICs se explicaría, por un lado, a la desidia de los docentes de aplicarlas y por otro lado a que se esfuerzan poco en exigir que se las utilice, por representar mayor esfuerzo y dedicación a su proceso de enseñanza, a pesar que la mayoría de estudiantes están familiarizados con las TICs y muchos de ellos la dominan, en desmedro de las actividades que les gusta realizar en los medios online.

Si bien es cierto que docentes y estudiantes tienen una actitud favorable hacia las TICs, esta es solo muy favorable para $22 \%$ de los estudiantes versus $29 \%$ de los docentes, a pesar que el mundo actual reclama una actitud muy favorable hacia las TICs. En este sentido, enfatiza Roquez ${ }^{\left({ }^{(8)}\right.}$ que el mayor acceso a la educación y a la cultura "cambia el modelo educativo del tradicional pasivo a otro activo, transforma el rol de profesor en facilitador, facilita la integración y la comunicación a los discapacitados, permite la educación e investigación a distancia interactiva y multimedia y globaliza el conocimiento de las diferencias culturales, sociales, lingüísticas, etc.".

En el estudio realizado por Área, Sanabria y González ${ }^{(9)}$ se evidenció que los estudiantes empezaban con unas expectativas altas sobre la docencia virtual, dado que les aportaría autonomía, flexibilidad y que aprenderían más. La UNESCO ${ }^{(5)}$ remarcaba que "Se reconoce que el uso de las TICs en educación puede ampliar el acceso a oportunidades de aprendizaje, mejorar sus logros de aprendizaje y calidad de la educación incorporando métodos avanzados de enseñanza, así como impulsar la reforma de los sistemas educativos".

La aparente desidia de los docentes a la utilización de las TICs estaría relacionada a la falta de tiempo, dado que tienen que trabajar en varias instituciones para poder subsistir ${ }^{(7)}$. Si se incrementara el número de docentes a tiempo completo podría aumentarse el uso de la TICs en el proceso educativo ${ }^{(7)}$. En este sentido, Muñoz-Rivas, Navarro y Ortega ${ }^{(10)}$ precisan que "En general, los estudiantes encuestados realizan un uso muy moderado de internet, dedicando un promedio semanal que va de 11 a 20 horas, lo que nos indicaría que no hay un uso abusivo o patológico cercano a la adicción, siendo un grupo reducido de jóvenes los que sí estarían próximos a este tipo de psicopatología."

En este sentido, la internet ha constituido una red social específica que puede afectar al estudiante tanto a nivel personal como académico, como lo acota Parra Castrillón (11). "Las redes son escenarios para la interacción social, definidas desde un intercambio dinámico entre personas en la web. Es tan avasallante su impacto, que para los nativos digitales su pertenencia a estas redes puede considerarse un asunto ya natural. Las cifras así lo revelan, pues el 97\% de los estudiantes han interactuado con Facebook, sin que esta sea la única red, pues en la web hay otras con buena representación". Marqués ${ }^{(12)}$ precisa que la "sociedad de la informa- 
ción en general y las nuevas tecnologías en particular inciden de manera significativa en todos los niveles del mundo educativo, de manera que para los estudiantes el cambio y el aprendizaje continuo para conocer las novedades que van surgiendo cada día es lo normal".

Para Luna, Urkijo, Caño y Muñiz ${ }^{(13)}$ "la introducción de cualquier tecnología de la información y comunicación en el contexto educativo pasa necesariamente tanto porque el profesorado tenga actitudes favorables hacia las mismas, como por una capacitación adecuada para su incorporación en su práctica profesional". Por tal razón, las universidades están en pleno proceso de tránsito de la cultura impresa a la digital, persistiendo aún los medios y métodos que sirvieron en un pasado inexistente a pesar que las nuevas tecnologías tratan de introducirse fuertemente en ella. Produciendo en los docentes incertidumbre, curiosidad y a veces rechazo ${ }^{(10)}$. La actitud de los docentes hacia las TICs en general fue menos favorable que la de los estudiantes, corroborando que los estudiantes por su calidad de nativos informáticos tienen una actitud más favorable. Finalmente, la satisfacción media global hacia las TICs fue mayor en los docentes que en los estudiantes.

\section{REFERENCIAS BIBLIOGRÁFICAS}

1. Carrera L. Análisis del perfil formativo en tecnologias de la información y comunicación del alumnado universitario y su adecuación a las necesidades empresariales en el ámbito de la CAPV. Tesis Doctoral. Disponible en: http://www.ehu.es/argitalpenak/images/stories/tesis/Ciencias_Sociales/ Analisis $\% 20$ del $\% 20$ perfil\%20formativo\%20en $\% 20$ tecnologia $\% 20$ de $\% 20$ la $\% 20$ informacion $\% 20 y \% 20$ comunicacion $\% 20$ del\%20alumnado\%20universitario.pdf.

2. Dussel I, Quevedo L. Educación y nuevas tecnologias: los desafios pedagógicos ante el mundo digital. Disponible en: http://virtualeduca.org/ifd/ pdf/ines-dussel.pdf.

3. Hernández D. Las actitudes de los docentes en el uso de las nuevas tecnologias de la información y comunicación de la I.E. Nº 88047 "Augusto Salazar Bondy" del distrito de Nuevo Chimbote. Disponible en: http://www.ongei.gob.pe/estudios/publica/ estudios/Lib5152/Libro.pdf.

4. Cabero J. La aplicación de las TIC, ¿esnobismo o necesidad educativa? Universidad de Sevilla. Disponible en: http://reddigital.cnice.mec.es/1/ cabero/01cabero_4.html.

5. UNESCO Las TIC en la Educación. Disponible en: http://www.unesco.org/new/es/unesco/themes/icts/ teacher-education/.

6. Marqués P. Nuevos instrumentos TIC para la educación. Como en los demás ámbitos de actividad humana, las TIC se convierten en un instrumento cada vez más indispensable en las instituciones educativas. 2000. Recuperado de http://www. peremarques.net/siyedu.htm.

7. Cabero J. Las necesidades de las TIC en el ámbito educativo: oportunidades, riesgos y necesidades. 2007. Disponible en: http://tecnologiaedu.us.es/ images/stories/jca51.pdf

8. Roquez A. Impactos de las tecnologias de información y comunicación en el Perú. Instituto Nacional de Estadistica e Informática. Lima. Perú. 2001.

9. Área M, Sanabria A, González M. Análisis de una experiencia de docencia universitaria semipre- sencial desde la perspectiva del alumnado. RIED. 2008;11(1):231-54

10. Muñóz-Rivas M, Navarro M, Ortega N. Patrones de uso de Internet en población universitaria española. Rev Adicciones. 2003;15(2):137-44.

11. Parra Castrillón E. Las redes sociales de Internet: también dentro de los hábitos de los estudiantes universitarios. Anagramas: Rumbos y sentidos de la comunicación. 2010;9(17):107-16.

12. Marqués P. Impacto de las TIC en el mundo educativo - funciones - niveles de integración y formas básicas de uso - ventajas e inconvenientes - ¿por qué tenemos que integrar las TIC? - entornos tecnológicos de E/A - modelos de aplicación práctica de las TIC - factores que inciden en su incorporación en la enseñanza - fuentes de información. 2011. Disponible en: http//www.peremarques.net.

13. Luna F, Urkijo M, Caño A, Muñiz M. Investigación: Integración de las TIC en centros de ESO. ISEI-IVEI. 2004.

14. Gómez J. El uso de las Tecnologías de Información y Comunicación (TIC) en los procesos de la educación básica primaria. Caso Enciclomedia. Tesis de Maestria en Ciencias Sociales. Facultad Latinoamericana de Ciencias Sociales. México. 2007.

Artículo recibido el 23 de abril de 2015 y aceptado para publicación el 23 de junio de 2015.

Fuentes de financiamiento: Propias.

Conflicto de intereses: Los autores expresan no tener algún conflicto de interés.

Correspondencia:

Dr. Luis Reátegui Guzmán

Facultad de Medicina Humana

Correo-e: reategui.asesores.salud@gmail.com 